\title{
Polaron mobility in oxygen-deficient and lithium-doped tungsten trioxide
}

\author{
N. Bondarenko, ${ }^{1}$ O. Eriksson, ${ }^{1}$ and N. V. Skorodumova ${ }^{1,2}$ \\ ${ }^{1}$ Division of Materials Theory, Department of Physics and Astronomy, Uppsala University, Box 516, 75121 Uppsala, Sweden \\ ${ }^{2}$ Multiscale Materials Modelling, Department of Materials Science and Engineering, Royal Institute of Technology, \\ SE-100 44 Stockholm, Sweden
}

(Received 25 June 2015; published 19 October 2015)

\begin{abstract}
Electron localization and polaron mobility in oxygen-deficient as well as Li-doped monoclinic tungsten trioxide have been studied in the adiabatic limit in the framework of density functional theory. We show that small polarons formed in the presence of oxygen vacancy prefer the bipolaronic $\mathrm{W}^{5+}-\mathrm{W}^{5+}$ configuration, whereas the $\mathrm{W}^{6+}-\mathrm{W}^{4+}$ configuration is found to be metastable. Our calculations suggest that bipolarons are tightly bound by the vacancy and therefore largely immobile. On the contrary, polarons formed as a result of Li intercalation can be mobile; the activation energy for polaron jumping in this case varies between 98 and $124 \mathrm{meV}$ depending on the crystallographic direction. The formation of $\mathrm{W}^{5+}-\mathrm{W}^{5+}$ bipolarons in $\mathrm{Li}-\mathrm{WO}_{3}$ is possible. When situated along [001] the bipolaronic configuration is $8 \mathrm{meV}$ lower in energy than two separate $\mathrm{W}^{5+}$ polarons.
\end{abstract}

DOI: 10.1103/PhysRevB.92.165119

PACS number(s): 71.38.-k, 78.20.Jq, 72.25.-b, 71.15.Mb

\section{INTRODUCTION}

The phenomenon of electrochromism is found in many materials such as organic compounds [1], polymer composites [2,3], and semiconductors [4]. One of the most studied materials of this class is tungsten trioxide due to a fortunate combination of its corrosion resistivity, sufficient light absorption, and satisfactory mechanical properties that make it an attractive material for advanced applications [5-8] such as "smart windows" technology $[9,10]$.

The first mention of $\mathrm{WO}_{3}$ coloration upon ion insertion dates back to the 19th century [11]. In the 20th century the interest in this material and its electrochromic properties revived and resulted in a large number of studies [12-15]. It is well known by now that "Prussian blue" coloration in the crystalline and amorphous phases of tungsten trioxide appears upon hydrogen or alkali-metal $(\mathrm{Li}, \mathrm{Na})$ intercalation $[4,9,10,16-18]$.

Tungsten trioxide is a wide band-gap semiconductor (2.6$3.0 \mathrm{eV}[17,19,20]$ ) with perovskite-like structure (see Fig. 1). The oxide shows rich structural polymorphism [21-24], whereby oxygen octahedrons surrounding tungsten atoms vary their orientations and shape depending both on temperature and pressure. Usually, at room temperature both $\delta-\mathrm{WO}_{3}$ (triclinic $P 1233-290 \mathrm{~K}$ ) and $\gamma-\mathrm{WO}_{3}$ (monoclinic $P 21 / n$ 290-350 K) phases coexist [17]. Depending on the method of preparation, samples can be characterized by various $\gamma / \delta$ ratios, microstructure, and defectiveness. Under certain conditions a more exotic hexagonal $\mathrm{h}-\mathrm{WO}_{3}$ phase $[25,26]$ or amorphous a- $\mathrm{WO}_{3}$ [16] phase can form. The structural variety of $\mathrm{WO}_{3}$ offers a range of optical characteristics that is important for applications relying on light absorption [27-29].

Alkali-metal intercalation can also cause structural transformation of $\mathrm{WO}_{3}$. For example, in the case of $\mathrm{Li}_{x} \mathrm{WO}_{3}$ several phase transitions from monoclinic to cubic symmetry with increasing Li content have been reported [30-32]. It has also been shown that for $\mathrm{Li}^{+}$intercalation the degree of coloration increases proportionally to the lithium concentration, whereas in the case of hydrogen insertion a saturation level of coloration is reached for a certain $\mathrm{H}^{+}$concentration [33]. Based on neutron diffraction data [34,35] this difference between $\mathrm{H}$ and $\mathrm{Li}$ has been explained by the formation of $\mathrm{O}-\mathrm{H}$ bonds upon hydrogen insertion. At the same time, alkali-metal ions show no tendency of binding to the oxygen atoms of the oxide matrix.

Substoichiometric $\mathrm{WO}_{3-x}$ also shows multifarious electrochromic behavior, demonstrating different degrees of coloration depending on the concentration of oxygen vacancies [36,37]. Thus, experiments clearly indicate that both doping $(\mathrm{H}, \mathrm{Li})$ and oxygen vacancies can influence $\mathrm{WO}_{3}$ optical properties [33,37-39].

Two general concepts of the coloration mechanism in tungsten oxide have been suggested: intervalence charge transfer [4] and polaron models [40,41], the latter being more commonly accepted. According to the polaron model the coloration is the result of photon absorption due to photon activated polaron hopping. A polaron can form in $\mathrm{WO}_{3}$ due to electron localization at the $d$ states of tungsten, thus turning $\mathrm{W}^{6+}$ into $\mathrm{W}^{5+}$ or $\mathrm{W}^{4+}$. Light absorption can lead to the activation of the following transitions: $\mathrm{W}_{\text {site } 1}^{5+}+\mathrm{W}_{\text {site } 2}^{6+} \stackrel{h v}{\rightarrow}$ $\mathrm{W}_{\text {site } 1}^{6+}+\mathrm{W}_{\text {site } 2}^{5+}$ or $\mathrm{W}_{\text {site } 1}^{5+}+\mathrm{W}_{\text {site } 2}^{5+} \stackrel{h v}{\rightarrow} \mathrm{W}_{\text {site } 1}^{6+}+\mathrm{W}_{\text {site } 2}^{4+}$. We notice that more complex configurations of localized charge can form, for example, two bound polarons at neighboring sites, $\mathrm{W}^{5+}-\mathrm{W}^{5+}$, also called bipolarons [see Figs. 1(b) and 1(c) for illustration].

The presence of different localized charge states in $\mathrm{WO}_{3}$ has been confirmed by numerous experiments [42-49]. The formation of polarons in this oxide has also been studied theoretically by semiempirical models [50,51] and more recently by $a b$ initio methods employing hybrid functionals [52]. More studies of the electronic and structural properties of perfect and defective $\mathrm{WO}_{3}$ can be found in Refs. [53-57]. Despite intensive research many questions regarding the properties of polarons in $\mathrm{WO}_{3}$ remain unclear. In this paper we address some of them; in particular, we study the formation and mobility of polarons in two cases: (1) in the presence of an oxygen vacancy and (2) in the presence of Li. We investigate the relative stability of polarons and bipolarons and calculate the energy barriers for polaron propagation in $\mathrm{WO}_{3}$. 

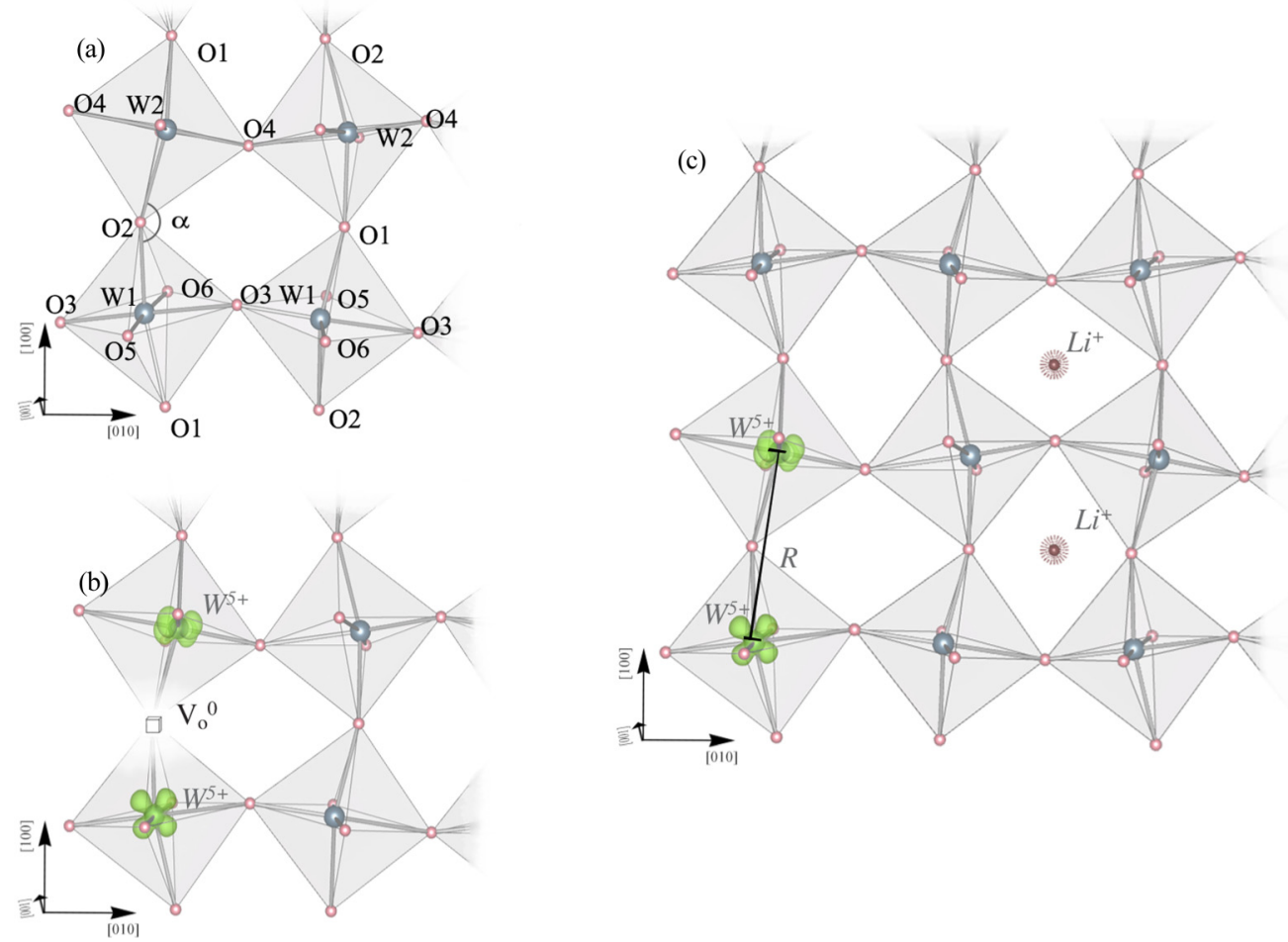

FIG. 1. (Color online) The structure of monoclinic $\gamma-\mathrm{WO}_{3}$ phase without defect (a), with an oxygen vacancy (b), and with two Li atoms (c). Charge distributions are shown for the polaronic $\mathrm{W}^{5+}$ states.

\section{DETAILS OF CALCULATIONS}

The presented calculations were performed in the framework of density functional theory (DFT) using the projector augmented-wave method, as implemented in VASP [58,59]. The DFT $+U[60]$ approach using generalized gradient approximation in the Perdew, Burke, and Ernzerhof [61] parametrization was used to account for the exchange-correlation interaction. The $U$ values $\left(U_{O_{p}}=9 \mathrm{eV}\right.$ and $\left.U_{W_{d}}=6 \mathrm{eV}\right)$ were determined using the linear response procedure implemented in the QUANTUM ESPRESSO package [62,63]. Hereafter we refer to this set of calculations as to DFT $+U_{W}, U_{O}$. We also performed the DFT $+U$ calculations with Hubbard- $U$ applied only to the $d$ states of $\mathrm{W}\left(\mathrm{DFT}+U_{\mathrm{W}}\right)$. Additionally, we used the screened hybrid functional of Heyd, Scuseria, and Ernzerhof (HSE06) [64,65] with the Coulomb potential separation parameter, $\omega$, of $0.2 \AA^{-1}$ and the $H F$ mixing constant of 0.25 .

Our DFT $+U$ calculations were performed for the $2 \times 2 \times$ 2 unit cell $(192 \mathrm{O}$ and $64 \mathrm{~W})$ and $2 \times 1 \times 1$ unit cell $(48 \mathrm{O}$ and $16 \mathrm{~W}$ ), both using a $\Gamma$ point centered $2 \times 2 \times 2 k$-point grid for the Brillouin zone integration. The $2 \times 1 \times 1$ unit cell was also used in the hybrid calculations. The following states were treated as valence states: $5 p^{6}, 5 d^{4}, 6 s^{2}(\mathrm{~W}), 2 s^{2}, 3 p^{4}(\mathrm{O})$, and $1 s^{1}, 2 p^{0}(\mathrm{Li})$. All the calculations were spin polarized. The cut-off energy was $650 \mathrm{eV}$.

The structure relaxation was done using the following scheme. First, we set up an initial supercell using the experimental parameters [23], and then we relaxed the structure in two steps: (1) first we fixed the atomic positions and relaxed only the cell parameters; (2) next we fixed the cell volume and relaxed the atomic positions. The latter were relaxed until the
Hellmann-Feynman forces acting on atoms became smaller than $10^{-4} \mathrm{eV} / \mathrm{A}$. We repeated this two-step relaxation routine a few times to ensure a stable result. We notice that during the relaxation we kept the symmetry of the lattice monoclinic. In order to localize the polaron in defective or Li-doped oxide, we introduced a local lattice deformation around a chosen $\mathrm{W}$ atom and then relaxed atomic positions in the supercell, while preserving equilibrium lattice parameters.

Here we study only uncharged oxygen vacancies, for which the vacancy formation energy, $E_{v}$, can be calculated as

$$
E_{v}=E_{\mathrm{W}_{x} \mathrm{O}_{3 x}}-E_{\mathrm{W}_{x} \mathrm{O}_{3 x-1}}-1 / 2 E_{\mathrm{O}_{2}} .
$$

The barriers for polaron transitions were calculated using both the linear interpolation scheme (LIS) as in our previous work [66] and the nudged elastic band method (NEB) [67]. NEB is an efficient method for studying atomic and molecular diffusion, phase transitions, chemical reactions, as well as polaronic transitions in oxides [67-71]. We used ten intermediate configurations in LIS and five images for NEB. Initial atomic positions for each NEB image were generated using a linear interpolation scheme.

\section{RESULTS AND DISCUSSION}

\section{A. Electron localization in oxygen-deficient $\boldsymbol{\gamma}-\mathrm{WO}_{3}$}

To properly model small polarons one needs a method adequately describing the on-site charge localization and lattice distortions around this site [66,72-75]. Electron localization in correlated $d$ - and $f$-metal oxides usually cannot be correctly described by standard DFT approximations, which due to the presence of the self-interaction term tend to overesti- 
mate the stability of the delocalized solution. The Coulomb interactions can be corrected, for example, by means of the Hubbard- $U$ parameter [76]. It can be treated as an adjustable parameter to better describe some experimental data [72] or it can be obtained from restricted ab initio calculations [63]. Traditionally in oxides Hubbard- $U$ is applied to the $d$ or $f$ states of cations; in some cases, however, the description of oxide property can be further improved by applying $U$ also to the $p$ states of oxygen [77-79]. Another way to cure the self-interaction problem of standard DFT is to employ the so-called hybrid functionals, where a certain amount of nonlocal Fock exchange is added [80]. Both approaches have successfully been used to describe electron localization or small polaron formation in different oxides [52,66,72-75,81]. Hybrid functionals are more computationally demanding than DFT $+U$ and therefore their usage is limited to rather small unit cells.

To find the optimal functional for the description of polarons in $\mathrm{WO}_{3}$ we have performed three sets of calculations using the $2 \times 1 \times 1$ unit cell (64 atoms), namely, DFT $+U_{\mathrm{W}}$, DFT $+U_{\mathrm{W}}, U_{\mathrm{O}}$ and HSE06 (see Sec. II for details). As a test case we considered an oxygen vacancy with two unpaired electrons localized on both sides of the vacancy in the $\mathrm{W}^{5+}-\mathrm{W}^{5+}$ configuration [Fig. 1(b)].

First, within the DFT $+U_{\mathrm{W}}$ approach we varied $U_{\mathrm{W}}$ from 2 to $12 \mathrm{eV}$ in the manner described in Ref. [66]. We analyzed the localization patterns for different values and found that for $U_{\text {eff }}=8 \mathrm{eV}$ and higher almost precisely one electron was localized at the $d$ orbitals of each of the two $\mathrm{W}^{5+}$ tungsten atoms. The local magnetic moment $\left(\mu_{\mathrm{W}}\right)$ for each $\mathrm{W}$ in this case was $0.96 \mu \mathrm{B}$. Next, we performed calculations using DFT $+U_{\mathrm{W}}, U_{\mathrm{O}}$. In this case the local magnetic moment due to the electron localization at the $\mathrm{W}_{d}$ states was $0.86 \mu \mathrm{B}$. Finally, our HSE06 calculation resulted in $\mu_{\mathrm{W}}=0.64 \mu \mathrm{B}$.

The density of states (DOSs) obtained in the three types of calculations are compared in Fig. 2. The band gaps are expectedly underestimated by both versions of the DFT $+U$ calculations: $E_{g}\left(\mathrm{DFT}+U_{\mathrm{W}}\right)=1.43 \mathrm{eV}$, and $E_{g}\left(\mathrm{DFT}+U_{\mathrm{W}}, U_{\mathrm{O}}\right)=1.64 \mathrm{eV}$ that should be compared to the experimental value of $2.8 \mathrm{eV}$ [17]. At the same time, HSE06 overestimates the gap: $E_{g}($ HSE06 $)=3.19 \mathrm{eV}$. In the case of DFT $+U_{\mathrm{W}}$ the localized polaronic $d$ states are situated at the upper edge of the valence band, whereas for HSE06 and DFT $+U_{\mathrm{W}}, U_{\mathrm{O}}$ these states are, respectively, 0.8 and $2.5 \mathrm{eV}$ above the valence band top. The position of the localized states with respect to the conduction band edge is similar for both approximations. We notice that the position of the polaronic peak in the gap obtained by HSE06 is in good agreement with previously reported B3LYP results [52].

The magnetic state of the obtained bipolaron is degenerate according to HSE06, whereas in the case of DFT $+U_{\mathrm{W}}, U_{\mathrm{O}}$ the antiferromagnetic coupling of local spins is favored by about $20 \mathrm{meV}$ over the ferromagnetic one. Using DFT $+U_{\mathrm{W}}, U_{\mathrm{O}}$ we also examined DOSs calculated for $\mathrm{Li}-\mathrm{WO}_{3}$ using the $2 \times 2 \times$ 2 unit cell and found them to be very similar to those calculated for the vacancy case. The only noticeable difference was a 40 $\mathrm{meV}$ shift of the polaronic peak down in energy.

The lattice parameters obtained using DFT $+U_{\mathrm{W}}, U_{\mathrm{O}}$ and HSE06 together with experimental data are shown in Table I. They demonstrate reasonable agreement with the HSE06

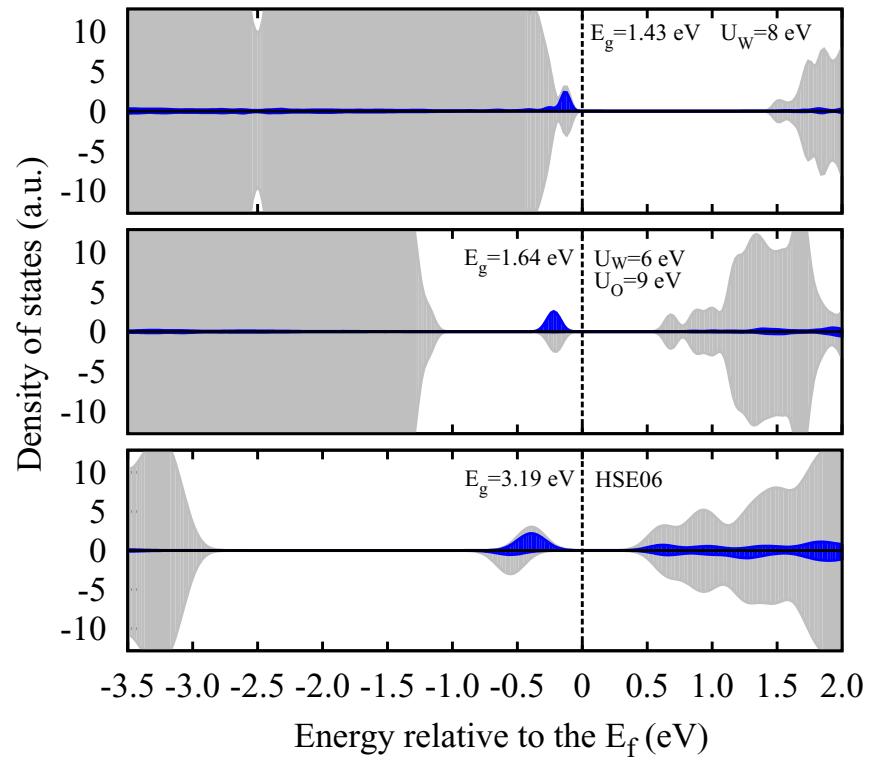

FIG. 2. (Color online) DOSs calculated for the $2 \times 1 \times 1$ supercell with an oxygen vacancy and associated $\mathrm{W}^{5+}-\mathrm{W}^{5+}$ bipolaron [see Fig. 1(b)]. The top, middle, and bottom panels present DOSs obtained with DFT $+U_{\mathrm{W}}$, DFT $+U_{\mathrm{W}}, U_{\mathrm{O}}$, and HSE06, respectively. The total DOSs are shown in gray, and the polaronic $\mathrm{W}_{d}$ states are in blue. The band gaps $E_{g}$ are given in the standard notation as a difference between the top of the valence band and the bottom of the conduction band.

values being a bit closer to the experimental parameters. Based on the analysis of DOSs, localization behavior, and structures obtained in the three sets of calculations we conclude that the DFT $+U_{\mathrm{W}}, U_{\mathrm{O}}$ approach is the most appropriate for studying polarons in $\mathrm{WO}_{3}$ as it shows good agreement with the results of HSE06 and, at the same time, allows us to treat large supercells. Therefore, further on we study polaron mobility in $\mathrm{WO}_{3}$ using the DFT $+U_{\mathrm{W}}, U_{\mathrm{O}}$ approach.

\section{B. Analysis of oxygen vacancy positions and vacancy trapped polarons}

The monoclinic phase of $\mathrm{WO}_{3}\left(2 c_{1} / n\right)$ has two tungsten and six oxygen nonequivalent atoms [23], as illustrated in Fig. 1(a). The structure is rather complex; each $\mathrm{O}$ atom has two neighboring $\mathrm{W}$ atoms but the $\mathrm{W}-\mathrm{O}-\mathrm{W}$ chains differ in all the directions. Along [100] we find ...-O1-W1-O2-W2-O1-... and ...-O2-W1-O1-W2-O2-... chains, along [001] ...-O6-W1-O5-W2-O6-... and ...-O5-W1-O6-W2-O5-...

TABLE I. The lattice parameters of the monoclinic cell obtained in the HSE06 and DFT $+U_{\mathrm{W}}, U_{\mathrm{O}}$ calculations together with experimental values [23]. In calculations angle $\beta$ was set to the experimental value $90.89^{\circ}$ [23].

\begin{tabular}{lccc}
\hline \hline $\begin{array}{l}\text { Lattice } \\
\text { parameters }(\AA)\end{array}$ & Experiment [23] & HSE06 & $\begin{array}{l}U\left(\mathrm{O}_{p}\right)=9 \mathrm{eV}, \\
U\left(\mathrm{~W}_{d}\right)=6 \mathrm{eV}\end{array}$ \\
\hline$a$ & 7.30 & 7.34 & 7.41 \\
$b$ & 7.54 & 7.58 & 7.65 \\
$c$ & 7.69 & 7.73 & 7.81 \\
\hline \hline
\end{tabular}


TABLE II. Bond distances and angles obtained for the monoclinic $\mathrm{WO}_{3}$ structure using DFT $+U_{\mathrm{W}}, U_{\mathrm{O}}$. The corresponding experimental values are shown in parentheses ([23]). The oxygen position notations are as shown in Fig. 1(a). The subscripts $x, y, z$ are added for convenience to indicate the orientation of the corresponding $\mathrm{W}^{5+}-\mathrm{W}^{5+}$ pair in the crystal.

\begin{tabular}{lccc}
\hline \hline Oxygen position & $\alpha(\mathrm{deg})$ & WO-bond1 $(\AA)$ & WO-bond2 (̊) \\
\hline $\mathrm{O} 1_{x},[100]$ & $160.1,(154.8)$ & $1.90,(1.89)$ & $1.89,(1.81)$ \\
$\mathrm{O}_{x},[100]$ & $160.7,(155.7)$ & $1.90,(1.95)$ & $1.89,(1.91)$ \\
$\mathrm{O}_{y},[010]$ & $163.6,(161.5)$ & $1.84,(1.82)$ & $2.03,(2.02)$ \\
$\mathrm{O}_{y},[010]$ & $163.4,(160.1)$ & $2.04,(2.06)$ & $1.84,(1.78)$ \\
$\mathrm{O}_{z},[001]$ & $170.5,(164.2)$ & $2.10,(2.16)$ & $1.80,(1.71)$ \\
$\mathrm{O}_{z},[001]$ & $169.7,(157.3)$ & $1.81,(1.81)$ & $2.09,(2.11)$ \\
\hline \hline
\end{tabular}

chains interconnected in the [010] direction by alternating ...W1-O3-W1-O3-W1-... and ...-W2-O4-W2-O4-W2-... chains [for notations see Fig. 1(a)]. The W-O bonds in all the directions have alternating shorter and longer distances and slightly different $\mathrm{O}-\mathrm{W}-\mathrm{O}$ angles, which are shown in Table II, both calculated with DFT $+U_{\mathrm{W}}, U_{\mathrm{O}}$ and from the experimental data [23].

Using the structure obtained in DFT $+U_{\mathrm{W}}, U_{\mathrm{O}}$ we calculated the vacancy formation energetics for the six oxygen positions [see Fig. 1(a)] and two bipolaronic configurations: one-centered $\mathrm{W}^{6+}-\mathrm{W}^{4+}$ bipolaron and two-centered $\mathrm{W}^{5+}-\mathrm{W}^{5+}$ bipolaron, where electrons are localized at each of the two opposing W sites [see Fig. 1(b)]. Both electronic configurations could be stabilized for all the vacancies. The results are presented in Fig. 3 and Table III. The lowest vacancy formation energy, $2.36 \mathrm{eV}$, is found for the oxygen vacancy at site $\mathrm{O}_{z}$ with bipolaronic configuration $\mathrm{W}^{5+}-\mathrm{W}^{5+}$ and the highest energy, $2.90 \mathrm{eV}$, for the $\mathrm{O} 1$ position in the $\mathrm{W}^{6+}-\mathrm{W}^{4+}$ configuration. The formation energies for the $\mathrm{W}^{6+}-\mathrm{W}^{4+}$ configurations are systematically higher than those for the $\mathrm{W}^{5+}-\mathrm{W}^{5+}$ ones (Table III). The vacancy formation

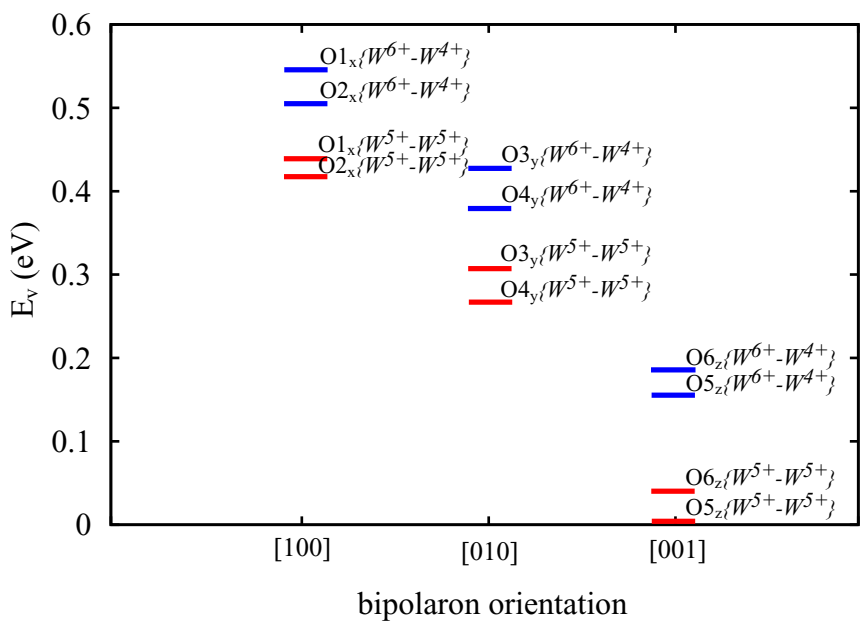

FIG. 3. (Color online) Vacancy formation energies for different vacancy positions and two electronic configurations: $\mathrm{W}^{6+}-\mathrm{W}^{4+}$ and $\mathrm{W}^{5+}-\mathrm{W}^{5+}$. The energies are shown with respect to the lowest formation energy, the one for the $5_{z}$ position, with the energy of $2.36 \mathrm{eV}$. See also Table III.
TABLE III. Vacancy formation energies, $E_{v}$, and relevant W$\mathrm{W}$ distances, $R$, for nonequivalent oxygen sites and $\mathrm{W}^{5+}-\mathrm{W}^{5+}$ [see Fig. 1(c)] and $\mathrm{W}^{6+}-\mathrm{W}^{4+}$ configurations. The numbers in parentheses show the corresponding W-W distances in the perfect $\mathrm{WO}_{3}$ cell.

\begin{tabular}{lcccc}
\hline \hline $\begin{array}{l}\text { Oxygen } \\
\text { position }\end{array}$ & $\begin{array}{c}E_{v}(\mathrm{eV}), \\
\mathrm{W}^{5+}-\mathrm{W}^{5+}\end{array}$ & $\begin{array}{c}E_{v}(\mathrm{eV}), \\
\mathrm{W}^{6+}-\mathrm{W}^{4+}\end{array}$ & $\begin{array}{c}R(\AA), \\
\mathrm{W}^{5+}-\mathrm{W}^{5+}\end{array}$ & $\begin{array}{c}R(\AA), \\
\mathrm{W}^{6+}-\mathrm{W}^{4+}\end{array}$ \\
\hline $\mathrm{O} 1_{x},[100]$ & 2.79 & 2.90 & $3.94,(3.72)$ & $3.87,(3.72)$ \\
$\mathrm{O} 2_{x},[100]$ & 2.77 & 2.86 & $3.92,(3.74)$ & $3.86,(3.74)$ \\
$\mathrm{O} 3_{y},[010]$ & 2.66 & 2.78 & $4.01,(3.83)$ & $3.90,(3.83)$ \\
$\mathrm{O} 4{ }_{y},[010]$ & 2.62 & 2.73 & $4.05,(3.84)$ & $3.92,(3.84)$ \\
$\mathrm{O}_{z},[001]$ & $\mathbf{2 . 3 6}$ & 2.51 & $\mathbf{4 . 3 5},(\mathbf{3 . 9 1})$ & $4.19,(3.91)$ \\
$\mathrm{O}_{z},[001]$ & 2.40 & 2.54 & $4.31,(3.89)$ & $4.14,(3.89)$ \\
\hline \hline
\end{tabular}

energy, $E_{v}$, depends on the orientation of the bipolaron in the lattice; $E_{v}$ averaged for each direction can be sorted in the following order: $E_{v}[100]>E_{v}[010]>E_{v}[001]$. These results are in agreement with previous studies carried out by standard DFT [57], where the oxygen atoms with the $\mathrm{W}-\mathrm{O}$ bonds along the [001] direction were indicated as favorable sites for vacancy formation. Using the B3LYP hybrid functional Wang et al. [52] also reported the formation of the $\mathrm{W}^{5+}-\mathrm{W}^{5+}$ configuration aligned with [001].

The data in Table III show that the vacancy formation energy decreases as the W-W distances increase and lowest $E_{v}$ corresponds to the largest W-W separation. The formation of bipolarons $\left(\mathrm{W}^{5+}-\mathrm{W}^{5+}\right)$ leads to a noticeable increase in the $\mathrm{W}-\mathrm{W}$ bond lengths due to Coulomb repulsion. These local lattice distortions are, however, different for different crystallographic directions. In the [100] and [010] directions the W-W distance increase is of about $0.20 \AA$, whereas for the $\mathrm{O}_{z}$ and $\mathrm{O}_{z}$ vacancy positions ([001]) it is 0.42 and $0.44 \AA$, respectively. We see a similar trend for the $\mathrm{W}^{6+}-\mathrm{W}^{4+}$ polaronic configurations (Table III), however, the changes of the W-W distance are smaller in this case. These results indicate certain softening of phonon modes along [001]. They also indicate that in the case of monoclinic $\mathrm{WO}_{3}$ the electronic contribution to the energy of vacancy formation dominates over the elastic contribution.

Our calculations suggest that the bipolaronic states associated with an oxygen vacancy are tightly trapped as all our attempts to localize charge away from the vacancy were unsuccessful. Therefore, we can expect that in the presence of oxygen vacancies bipolarons are immobilized in the lattice, however, the transitions between vacancy bound $\mathrm{W}^{5+}-\mathrm{W}^{5+}$ and $\mathrm{W}^{6+}-\mathrm{W}^{4+}$ could still contribute to the coloration effect in $\mathrm{WO}_{3}$. To study the transition between these two configurations we chose oxygen vacancies with lowest $E_{v}$ in each crystallographic direction, namely, $\mathrm{O} 2_{x}$, $\mathrm{O} 4_{y}$, and $\mathrm{O}_{z}$. Our results, presented in Fig. 4, show that the transition barriers vary from 150 to $230 \mathrm{meV}$, being the smallest for the [100] direction and largest for [001]. This result is in agreement with similar trends shown by the energy differences between the $\mathrm{W}^{5+}-\mathrm{W}^{5+}$ and $\mathrm{W}^{6+}-\mathrm{W}^{4+}$ configurations: $\mathrm{O} 2_{x},(90 \mathrm{meV})<\mathrm{O} 4_{y},(110 \mathrm{meV})<O 5_{z},(150 \mathrm{meV})$ and the W-W bond length differences: $\mathrm{O} 2_{x},(0.06 \AA)<$ $\mathrm{O} 4 y,(0.13 \AA)<O 5_{z},(0.16 \AA) x$. We also notice that the NEB 


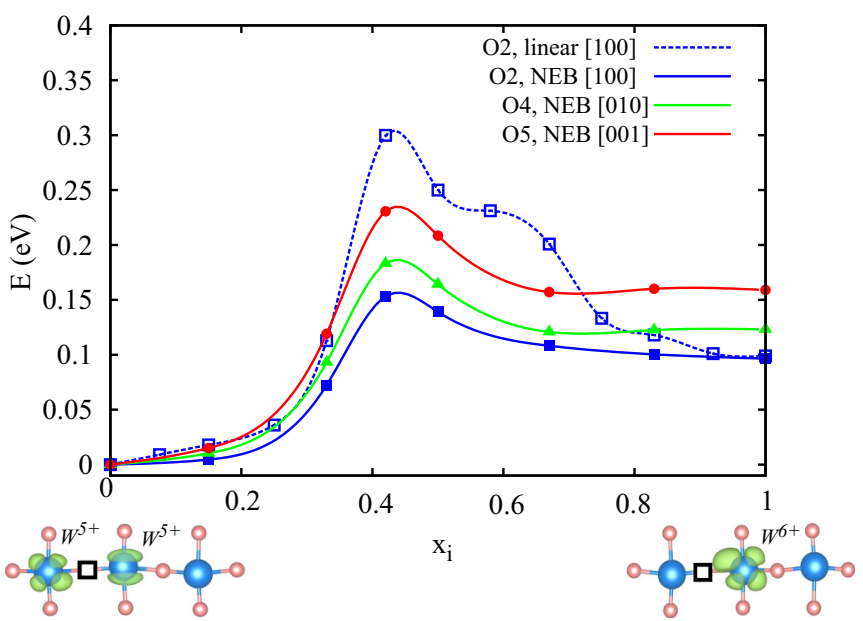

FIG. 4. (Color online) Energies along the transition path between the $\mathrm{W}^{5+}-\mathrm{W}^{5+}$ and $\mathrm{W}^{6+}-\mathrm{W}^{4+}$ configurations for three vacancies $\left(\mathrm{O} 2_{x}\right.$, $\mathrm{O}_{y}$, and $\mathrm{O}_{z}$ ) as estimated by NEB. The energies are given with respect to that of $\mathrm{W}^{5+}-\mathrm{W}^{5+}$ in the corresponding direction. For comparison for $\mathrm{O5}_{z}$ we show the results obtained by the LIS [66]. Charge distributions corresponding to the initial $x=0\left(\mathrm{~W}^{5+}-\mathrm{W}^{5+}\right)$ and final $x=1\left(\mathrm{~W}^{4+}\right)$ transition points are shown for illustration.

relaxation results in lower barriers compared to those obtained with LIS (Fig. 4). This is understandable as NEB allows one to optimize structures along the transition path, whereas LIS uses static configurations.

Lattice distortions around polarons and especially bipolarons can be quite substantial reaching far beyond first coordination shells. In the case studied here we find that the local distortions around the bipolaron-vacancy complex spreads as far as $10-11 \AA$ in the direction of the $\mathrm{W}^{5+}-\mathrm{W}^{5+}$ pair alignment. The $2 \times 2 \times 2$ cell used in our calculations is big enough to accommodate such a defect. Our results, however, highlight the importance of using large unit cells in modeling polarons and bipolarons at least if one wishes to study isolated quasiparticles of the sort.

\section{Polaron formation and transitions in Li-doped monoclinic $\gamma$ phase}

Polarons can also form in the presence of impurities, dopants, or due to electron injection. Here we report our results of the polaron formation and mobility in Li-doped monoclinic $\mathrm{WO}_{3}$. First we consider one lithium atom situated in an octapore in the supercell of 256 atoms, i.e., $\mathrm{WO}_{3} \mathrm{Li}_{0.016}$. This is a realistic concentration often studied in experiment [82]. After the relaxation $\mathrm{Li}$ stayed close to the octapore center [Fig. 1(c)], no bond formation with the surrounding oxygen atoms was detected. It was possible to localize the $\mathrm{W}^{5+}$ polaron at different tungsten sites. The association energy of $\mathrm{Li}^{+}$and $\mathrm{W}^{5+}$ is of the order of $30 \mathrm{meV}$, which was estimated by calculating the energies of $\mathrm{Li}^{+}-\mathrm{W}^{5+}$ separated by 3.92 and $7.71 \AA$ A.

Further, to study polaron mobility we used tungsten sites away from $\mathrm{Li}(\sim 6-8 \AA)$ simulating a free polaron propagation in the $\mathrm{WO}_{3}$ matrix. Figure 5 shows the energy barriers for polaron jumping between two neighboring $\mathrm{W}$ sites in the

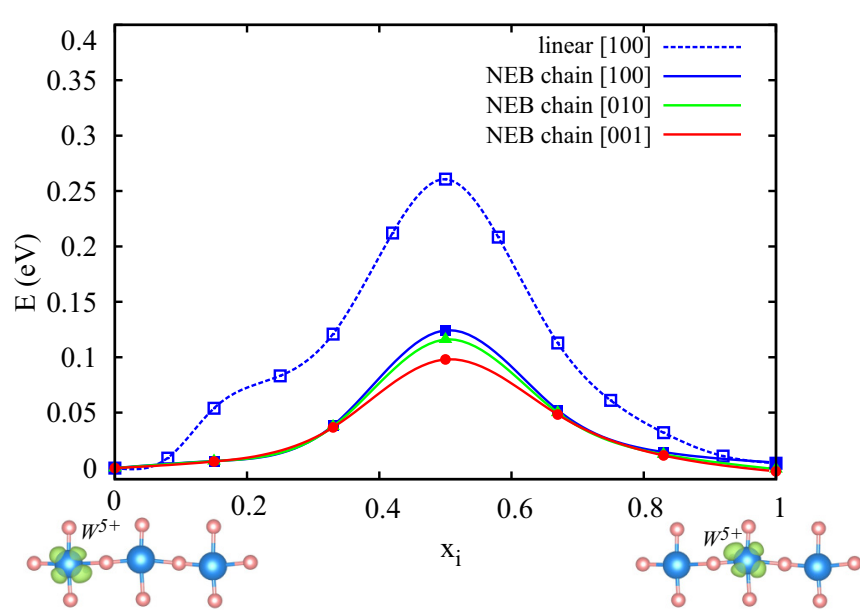

FIG. 5. (Color online) Energies along the transition paths of $\mathrm{W}^{5+}$ from one tungsten site to the other in the case of Li-doped $\gamma$ phase. The energies are given with respect to the energy of $\mathrm{W}^{5+}$. The solid lines show the NEB energies for all three crystallographic directions. For comparison, for [100] we also show the energies obtained in LIS calculations (dashed line). Charge distributions for $\mathrm{W}^{5+}$ at the initial $x=0$ and final $x=1$ transition points are shown for illustration.

three crystallographic directions. As expected, the LIS barriers are again higher than those obtained using NEB. The NEB barriers vary from 98 for [001] to $124 \mathrm{meV}$ for [100]. These values are in surprisingly close agreement with the activation energy for polaron hopping estimated from basic models using experimental information, $180 \mathrm{meV}$ [82].

If the concentration of lithium ions and polarons increases the number of possible configurations of localized charges becomes larger. We study the possibility of a bipolaron formation by considering two $\mathrm{Li}$ atoms in the 256 atom $\mathrm{WO}_{3}$ supercell $\left(\mathrm{WO}_{3} \mathrm{Li}_{0.032}\right)$. Next to the bipolaronic $\mathrm{W}^{5+}-\mathrm{W}^{5+}$ configurations we also consider the formation of two separate $\mathrm{W}^{5+}$ polarons and one $\mathrm{W}^{4+}$. In Fig. 6 we show the energies for different electronic configurations and transitions between them obtained using NEB. We start from the situation when both electrons donated by the two Li atoms are delocalized in the oxide matrix ( $x=0$, Fig. 6 ), then we localize one $\mathrm{W}^{5+}$ polaron that happens without any barrier $(x=2$, Fig. 6). After that we localize the second electron at the next nearest $(\mathrm{NN}) \mathrm{W}$ site from the first $\mathrm{W}^{5+}$, which neither involves any barrier $(x=3$, Fig. 6). The energies of such NN configurations are very close to each other for all the orientations (Fig. 6). Next we model a transition of one $\mathrm{W}^{5+}$ from the $\mathrm{NN}$ site to the nearest (N) W site, thus forming a bipolaron along the [100], [010], and [001] directions ( $x=3 \rightarrow x=5$, Fig. 6). This transition involves a barrier of about $90 \mathrm{meV}$. The resulting bipolaron situated along [001] has the lowest energy not only among the bipolarons but among all the considered configurations (Fig. 6). The [001] bipolaron is $8 \mathrm{meV}$ lower in energy than the [001] NN configuration. At the same time, the energies of bipolarons oriented along [100] and [010] are about 20 meV higher than those of the NN configurations. We notice that the localization of two electrons at one tungsten site $\left(\mathrm{W}^{4+}\right)$ is energetically unfavorable as this state lies more than $300 \mathrm{meV}$ above any $\mathrm{W}^{5+}-\mathrm{W}^{5+}$ configuration. Therefore, our results suggest that in $\mathrm{Li}$-doped $\mathrm{WO}_{3}$ one can expect 


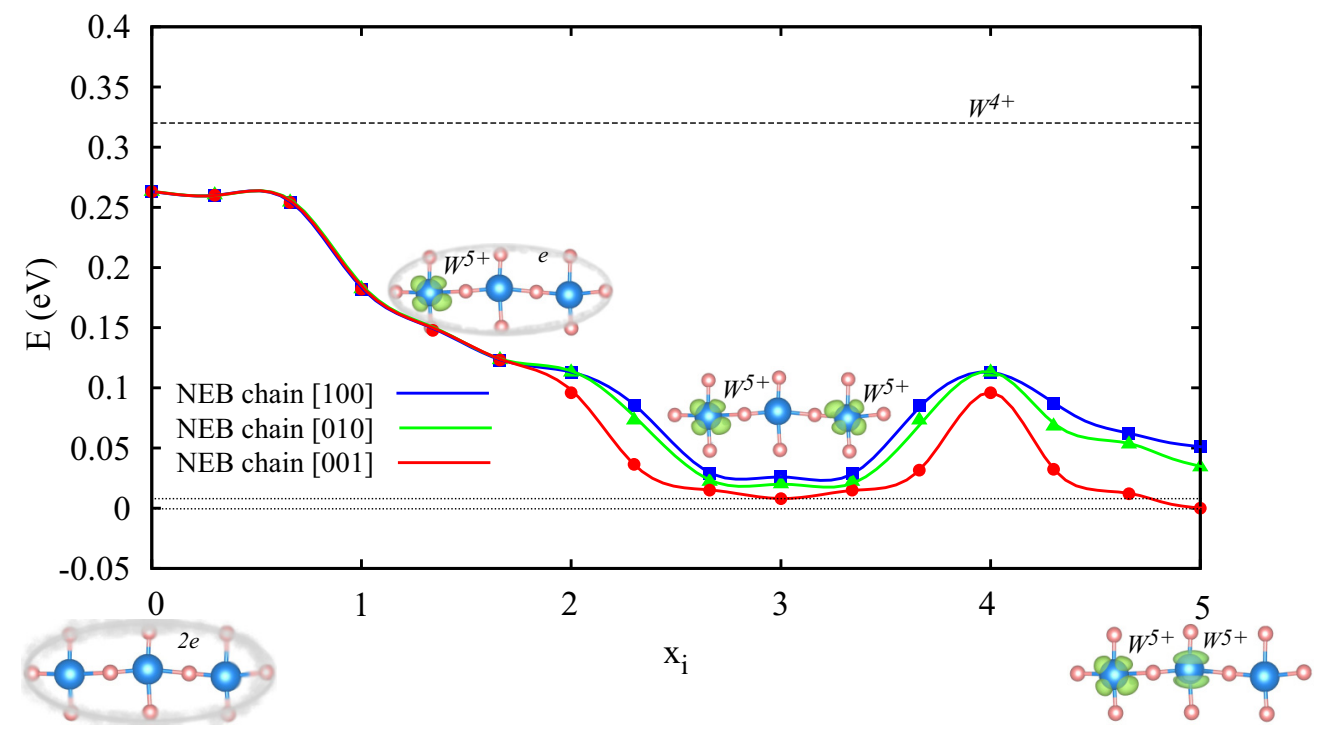

FIG. 6. (Color online) Energies along the transition paths calculated using NEB for the case of two intercalated Li atoms [Fig. 1(c)]. The energies are shown with respect to the lowest energy configuration $\mathrm{W}^{5+}-\mathrm{W}^{5+}$ situated along [001]. The following polaronic transitions are shown: from $x=0$ (two delocalized electrons) $\rightarrow x=2$ (one electron localized as $\mathrm{W}^{5+}$, the other is delocalized) then $\rightarrow x=3$ (two $\mathrm{W}^{5+}$ as next nearest neighbors) and further $\rightarrow x=5\left(\mathrm{~W}^{5+}-\mathrm{W}^{5+}\right.$ bipolaron configurations). These transitions are calculated along [100], [010], and [001]. The energy of the $\mathrm{W}^{4+}$ configuration is marked by the dashed line. Two dotted lines indicate the energy difference between the $\mathrm{W}^{5+}-\mathrm{W}^{5+}$ bipolaron and NN configuration situated along the [001] direction. Charge distributions for the $\mathrm{W}^{5+}$ polarons at $x=2,3$, and 5 are shown for illustration.

the formation of $\mathrm{W}^{5+}$ polarons and $\mathrm{W}^{5+}-\mathrm{W}^{5+}$ bipolarons, whereas the presence of the $\mathrm{W}^{4+}$ state, often detected in experiments $[29,33,36,46,48,49]$, most likely can be attributed to oxygen vacancies, around which the $\mathrm{W}^{4+}$ state is found to be metastable.

\section{CONCLUSION}

We have studied the applicability of HSE06 and two $\mathrm{DFT}+U$ approaches to modeling of polarons in $\mathrm{WO}_{3}$. We have shown that the DFT $+U$ approach with two Hubbard- $U$ parameters determined and applied simultaneously to the $d-\mathrm{W}\left(U_{\mathrm{W}}=6 \mathrm{eV}\right)$ and $p-\mathrm{O}\left(U_{\mathrm{O}}=9 \mathrm{eV}\right)$ states provides a proper description of polaron formation and transitions in $\mathrm{WO}_{3}$. At the same time, this method allows us to calculate large unit cells necessary for adequate modeling of polarons and bipolarons. Using this approach we have studied the vacancy energetics for the six nonequivalent oxygen positions and two electronic configurations: $\mathrm{W}^{5+}-\mathrm{W}^{5+}$ and $\mathrm{W}^{6+}-\mathrm{W}^{4+}$. The $\mathrm{W}^{5+}-\mathrm{W}^{5+}$ bipolarons situated along [001] are the most favorable electronic configurations around vacancies. We have also studied polaron and bipolaron formation and energetics for $\mathrm{Li}$-doped $\mathrm{WO}_{3}$. In this case the $\mathrm{W}^{5+}-\mathrm{W}^{5+}$ bipolaronic configuration aligned with [001] has again the lowest energy but winning only $8 \mathrm{meV}$ over two separated $\mathrm{W}^{5+}$. Our results suggest that the polarons formed due to oxygen vacancies are immobile, at the same time, the $\mathrm{W}^{4+}$ state is metastable and the $\mathrm{W}^{5+}-\mathrm{W}^{5+}$ to $\mathrm{W}^{6+}-\mathrm{W}^{4+}$ transition is possible with a barrier of $150 \mathrm{meV}$. On the contrary, polarons formed in $\mathrm{Li}$ doped tungsten oxide are mobile with the minimum activation energy $(98 \mathrm{meV})$ in the [001] direction. The $\mathrm{W}^{4+}$ state is $300 \mathrm{meV}$ higher in energy than any studied $\mathrm{W}^{5+}$ configuration and, therefore, $\mathrm{W}^{4+}$ is unlikely to form in perfect $\mathrm{Li}-\mathrm{WO}_{3}$ without the presence of vacancies or similar structural defects.

\section{ACKNOWLEDGMENTS}

We acknowledge the financial support by the Swedish Energy Agency (Energimyndigheten, STEM) eSSENCE, STANDUPP, and the Swedish Research Council (Vetenskapsrådet). Supercomputer time was granted by the Swedish National Infrastructure for Computing (SNIC). N.B. is grateful to National Supercomputer Centre (NSC) for provided support. O.E. acknowledges, in addition, the KAW. Authors are thankful to C.-G. Granqvist and G. Niklasson for valuable discussions.
[1] R. J. Mortimer, Electrochim. Acta 44, 2971 (1999).

[2] F. Carpi and D. De Rossi, IEEE Trans. Inf. Technol. Biomed. 9, 295 (2005).

[3] J. Heinze, Electronically conducting polymers, Top. Curr. Chem. 152 (1990).

[4] C. G. Granqvist, Handbook of Inorganic Electrochromic Materials (Elsevier New York, 1995).
[5] G. Hodes, D. Cahen, and J. Manassen, Nature (London) 260, 312 (1976).

[6] A. Azens, A. Hjelm, D. Le Bellac, C. G. Granqvista, J. Barczynskab, E. Pentjuss, J. Gabrusenoks, and J. M. Willsd, Solid State Ionics 86, 943 (1996).

[7] H. Watanabe, K. Fujikata, Y. Oakib, and H. Imai, Chem. Commun. 49, 8477 (2013). 
[8] H. Zheng, J. Z. Ou, M. S. Strano, R. B. Kaner, A. Mitchell, and K. Kalantar-Zadeh, Adv. Funct. Mater. 21, 2175 (2011).

[9] C. G. Granqvist, Adv. Sci. Technol. 55, 205 (2008).

[10] J. S. E. M. Svensson and C. G. Granqvist, Sol. Energ. Mater. 11, 29 (1984).

[11] J. J. Berzelius, Afh. Fys., Kemi Mineral. 4, 293 (1815).

[12] S. K. Deb, Appl. Opt. 3, 192 (1969).

[13] B. W. Faughnan, R. S. Crandall, and M. A. Lampert, Appl. Phys. Lett. 27, 275 (1975).

[14] B. Reichman and A. J. Bard, J. Electrochem. Soc. 126, 583 (1979).

[15] C. G. Granqvist, Sol. Energy Mater. Sol. Cells. 99, 1 (2012).

[16] J.-G. Zhang, D. K. Benson, C. E. Tracy, S. K. Deb, A. W. Czandema, and C. Bechinger, Chromic mechanism in amorphous $\mathrm{WO}_{3}$ films, 190th Electrochemical Society Meeting, 1996 (unpublished).

[17] M. B. Johansson, G. A. Niklasson, and L. J. Österlund, J. Mater. Res. 27, 3130 (2012).

[18] S. Raj, H. Matsui, S. Souma, T. Sato, T. Takahashi, A. Chakraborty, D. D. Sarma, P. Mahadevan, S. Oishi, W. H. McCarroll, and M. Greenblatt, Phys. Rev. B 75, 155116 (2007).

[19] R. G. Bamwenda, K. Sayama, and H. Arakawa, J. Photochem. Photobiol. A 122, 175 (1999).

[20] C. G. Granqvist, Sol. Energy Mater. Sol. Cells 60, 201 (2000).

[21] S. Tanisaki, J. Phys. Soc. Jpn. 15, 566 (1960).

[22] R. Diehl and G. Brandt, Acta Crystallogr., Sect. B: Struct. Sci., Cryst. Eng. Mater. 34, 1105 (1978).

[23] P. W. Woodward, A. W. Sleight, and T. J. Vogt, J. Phys. Chem. Solids 56, 1305 (1995).

[24] C. J. Howard, V. Luca, and K. S. Knight, J. Phys.: Condens. Matter 14, 377 (2002).

[25] B. Gerand, G. Nowogrocki, J. Guenot, and M. Figlarz, J. Solid State Chem. 29, 429 (1979).

[26] O. Yu. Khyzhun, Yu. M. Solonin, and V. D. Dobrovolsky, J. Alloy. Compd. 320, 1 (2001).

[27] K. Miyake, H. Kaneko, M. Sano, and N. Suedomi, J. Appl. Phys. 55, 2747 (1984).

[28] A. Antonaia, M. L. Addonizio, C. Minarini, T. Polichetti, and M. Vittori-Antisari, Electrochim. Acta 46, 2221 (2001).

[29] S. S. Sun and P. H. Holloway, J. Vac. Sci. Technol., A 2, 336 (1984).

[30] K. R. Dey, C. H. Ruscher, Th. M. Gesing, and A. Hussain, Mater. Res. Bull. 42, 591 (2007).

[31] Q. Zhong, J. R. Dahn, and K. Colbow, Phys. Rev. B 46, 2554 (1992).

[32] S. Lee, M. J. Seong, H. M. Cheong, E. Ozkan, E. C. Tracy, and S. K. Deb, Solid State Ionics 156, 447 (2003).

[33] P. Gerard, A. Deneuville, and R. Courths, Thin Solid Films 71, 221 (1980).

[34] W. Wright, J. Solid State Chem. 20, 89 (1977).

[35] P. J. Wiseman and P. G. Dickens, J. Solid State Chem. 6, 374 (1973).

[36] P. Uppachai, V. Harnchana, S. Pimanpang, V. Amornkitbamrung, A. P. Brown, and R. M. D. Brydson, Electrochim. Acta 145, 27 (2014).

[37] T. Yoshimura, J. Appl. Phys. 57, 911 (1985).

[38] A. Deneuville and P. Gerard, J. Electron. Mater. 7, 559 (1978).

[39] O. Pyper, A. Kaschnerb, and C. Thomsen, Sol. Energy Mater. Sol. Cells 71, 511 (2002).
[40] O. F. Schirmer, V. Wittwer, G. Baur, and G. Brandt, J. Electrochem. Soc. 124, 749 (1977).

[41] E. Salje, Opt. Commun. 24, 231 (1978).

[42] L. Berggren, A. Azens, and G. A. Niklasson, J. Appl. Phys. 90, 1860 (2001).

[43] M. F. Saenger, T. Höing, T. Hofmann, and M. Schubert, Phys. Status Solidi A 4, 914 (2008).

[44] S. Hashimoto and H. Matsuoka, J. Appl. Phys. 69, 933 (1991).

[45] H. Chen, N. Xu, S. Deng, J. Zhou, Z. Li, H. Ren, J. Chen, and J. She, J. Appl. Phys. 101, 114303 (2007).

[46] M. M. Johansson, B. Zietz, G. A. Niklasson, and L. Österlund, J. Appl. Phys. 115, 213510 (2014).

[47] G. A. Niklarsson, A. Norling, G. Possnert, and L. Berggren, J. Phys.: Conf. Ser. 100, 082023 (2008).

[48] E. Ozkan, S. Lee, C. E. Tracya, J. R. Pittsa, and S. K. Deb, Sol. Energy Mater. Sol. Cells 79, 439 (2003).

[49] G. Leftheriotis S. Papaefthimioua, P. Yianoulisa, and A. Siokoub, Thin Solid Films 384, 298 (2001).

[50] E. Iguchi and H. Miyaghi, J. Phys. Chem. Solids. 54, 403 (1993).

[51] A. Stashans and S. Lunell, Int. J. Quantum Chem. 63, 729 (1997).

[52] F. Wang, C. Di Valentin, and G. Pacchioni, Phys. Rev. B 84, 073103 (2011).

[53] G. A. de Wijs and R. A. de Groot, Phys. Rev. B 60, 16463 (1999).

[54] D. B. Migas, V. L. Shaposhnikov, V. N. Rodin, and V. E. Borisenko, Appl. Phys. 108, 093713 (2010).

[55] N. L. Heda and B. L. Ahuja, Comput. Mater. Sci. 72, 49 (2013).

[56] R. Chatten, A. V. Chadwick, A. Rougier, and P. J. D. Lindan, J. Phys. Chem. 109, 3146 (2005).

[57] C. Lambert-Mauriata and V. Oison, Surf. Sci. 606, 40 (2012).

[58] G. Kresse and J. Hafner, Phys. Rev. B 47, 558 (1993); 49, 14251 (1994).

[59] G. Kresse and J. Furthmüller, Comput. Mater. Sci. 6, 15 (1996); Phys. Rev. B 54, 11169 (1996).

[60] S. L. Dudarev, G. A. Botton, S. Y. Savrasov, C. J. Humphreys, and A. P. Sutton, Phys. Rev. B 57, 1505 (1998).

[61] J. P. Perdew, K. Burke, and M. Ernzerhof, Phys. Rev. Lett. 77, 3865 (1996).

[62] P. Giannozzi, S. Baroni, N. Bonini, M. Calandra, R. Car, C. Cavazzoni, D. Ceresoli, G. L. Chiarotti, M. Cococcioni, I. Dabo, A. Dal Corso, S. de Gironcoli, S. Fabris, G. Fratesi, R. Gebauer, U. Gerstmann, C. Gougoussis, A. Kokalj, M. Lazzeri, L. Martin-Samos, N. Marzari, F. Mauri, R. Mazzarello, S. Paolini, A. Pasquarello, L. Paulatto, C. Sbraccia, S. Scandolo, G. Sclauzero, A. P. Seitsonen, A. Smogunov, P. Umari, and R. M. Wentzcovitch, J. Phys.: Condens. Matter 21, 395502 (2009).

[63] M. Cococcioni and S. de Gironcoli, Phys. Rev. B 71, 035105 (2005).

[64] J. Heyd, G. E. Scuseria, and M. Ernzerhof, J. Chem. Phys. 118, 8207 (2003).

[65] J. Heyd and G. E. Scuseria, J. Chem. Phys. 120, 7274 (2004).

[66] N. Bondarenko, O. Eriksson, and N. V. Skorodumova, Phys. Rev. B 89, 125118 (2014).

[67] G. Henkelman and H. Jonsson, J. Chem. Phys. 113, 9901 (2000).

[68] D. Sheppard, P. H. Xiao, W. Chemelewski, D. D. Johnson, and G. Henkelman, J. Chem. Phys. 136, 74103 (2012).

[69] N. Gonzalez-Garcia, J. Pu, Á. Gonzalez-Lafont, J. M. Lluch, and D. G. Truhlar, Chem. Theory Comput. 2, 895 (2006). 
[70] L. Xie, H. Y. Liu, and W. T. Yang, J. Chem. Phys. 120, 8039 (2004).

[71] S. P. Ong, Y. Mo, and G. Ceder, Phys. Rev. B 85, 081105 (2012).

[72] D. A. Andersson, S. I. Simak, B. Johansson, I. A. Abrikosov, and N. V. Skorodumova, Phys. Rev. B 75, 035109 (2007).

[73] S. Arapan, S. I. Simak, and N. V. Skorodumova, Phys. Rev. B 91, 125108 (2015).

[74] T. Maxisch, F. Zhou, and G. Ceder, Phys. Rev. B 73, 104301 (2006).

[75] A. Droghetti, C. D. Pemmaraju, and S. Sanvito, Phys. Rev. B 81, 092403 (2010).
[76] V. I. Anisimov, J. Zaanen, and O. K. Andersen, Phys. Rev. B 44, 943 (1991).

[77] N. A. Deskins and M. Dupuis, J. Phys. Chem. C 113, 346 (2009).

[78] X. Ma, Y. Wu, Y. Lv, and Y. Zhu, J. Phys. Chem. C 117, 26029 (2013).

[79] P. Erhart, A. Klein, D. Aberg, and B. Sadigh, Phys. Rev. B 90, 035204 (2014).

[80] A. D. Becke, J. Chem. Phys. 98, 5648 (1993).

[81] C. Franchini, G. Kresse, and R. Podloucky, Phys. Rev. Lett. 102, 256402 (2009).

[82] A. L. Larsson, B. E. Sernelius, and G. A. Niklasson, Solid State Ionics 165, 35 (2003). 